\title{
PReaCH: A Fast Lightweight Reachability Index using Pruning and Contraction Hierarchies
}

\author{
Florian Merz and Peter Sanders \\ Karlsruhe Institute of Technology, Karlsruhe, Germany \\ sanders@kit.edu,flomerz@gmail.com
}

October 18, 2018

\begin{abstract}
We develop the data structure PReaCH (for Pruned Reachability Contraction Hierarchies) which supports reachability queries in a directed graph, i.e., it supports queries that ask whether two nodes in the graph are connected by a directed path. PReaCH adapts the contraction hierarchy speedup techniques for shortest path queries to the reachability setting. The resulting approach is surprisingly simple and guarantees linear space and near linear preprocessing time. Orthogonally to that, we improve existing pruning techniques for the search by gathering more information from a single DFS-traversal of the graph. PReaCH-indices significantly outperform previous data structures with comparable preprocessing cost. Methods with faster queries need significantly more preprocessing time in particular for the most difficult instances.
\end{abstract}

\section{Introduction}

Many applications are modelled using graphs of some kind. One of the most fundamental questions one may ask about a graph is whether there is a path between two given nodes. For example, in a graph modelling the hierarchy of a company one might ask whether one person is subordinate to another. In a network of papers with links expressing citations, one might ask whether one paper is based on another paper in some, possibly indirect, way. Important applications are semantic networks / RDF graphs [22] and XML databases [11]. Applications in bioinformatics databases include protein-protein interactions [22], metabolic networks [21], and gene regulatory networks [14]. Due to these manyfold applications and since reachability queries are very expensive in classical data bases, the reachability problem has obtained a lot of attention in the database community.

Reachability queries can be answered in linear time using any kind of graph exploration, e.g., by breadth first search. However, for many applications this is too slow since a large number of queries has to be answered. Assuming that the graph changes rarely, one can afford 
to do some preprocessing, i.e., we compute a data structure $I$ that helps to accelerate later queries. I can be viewed as an index data structure. When comparing such preprocessing approaches one faces a tradeoff between at least three criteria - preprocessing time, the space needed for the index, and the query time. For example, the above query by BFS approach has zero preprocessing costs yet requires linear query time. On the other hand, precomputing all possible answers needs space quadratic in the number of nodes but allows constant time queries. Clearly, compromises are relevant here.

Our starting point was the idea to transfer the rapid recent progress on speedup techniques for route planning to reachability indices. We settled on Contraction Hierarchies (CHs) [12] because they are one of the most successful such techniques and because we found an adaptation to the reachability problem with surprisingly low preprocessing time. In comparison, other successful techniques such as arc flags, landmark $A^{*}$, transit node routing, customizable route planning or hub labelling (see [2] for a recent survey) seem to be inferior at least with respect to guarantees on preprocessing time. In Section 3.1 it turns out that Reachability Contraction Hierarchies ( $R C H s$ ) are much simpler than shortest path $\mathrm{CHs}$ and guarantee near linear preprocessing time and linear space consumption. During preprocessing, RCHs just repeatedly remove nodes with in-degree or out-degree zero. Edges leaving nodes with in-degree (out-degree) zero are marked for forward (backward) exploration. Queries are based on bidirectional graph exploration. Forward (backward) exploration only has to consider edges marked as forward (backward) during preprocessing. This can lead to dramatic speedups since the average branching factor of the graph exploration is halved. In contrast to RCHs, shortest path $\mathrm{CHs}$ need to insert additional shortcut edges during construction which may lead to quadratic space and cubic preprocessing for graphs that do not have very pronounced hierarchical properties such as road networks.

An equally important ingredient of PReaCH is a suite of heuristics for pruning graph exploration during a query. We adopt and improve techniques from GRAIL [34]: Topological levels are essentially a compressed form of a topological ordering. When the level of a node $v$ is larger or equal to the level of a node $t$ then there certainly is no path from $v$ to $t$. This information can be used for both forward and backward search and we can calculate different topological levels to allow further pruning, see Section 3.2 for more details.

More sophisticated pruning rules are derived from a DFS-numbering of the nodes. For each node $v$, we identify two ( full) ranges of DFS numbers of nodes that are reachable from $v$ and three (empty) ranges of DFS numbers that are not reachable from $v$. During a query, full ranges can be used to stop the search completely while the empty ranges can prune the search as in topological levels. All these ranges can be derived from a single DFS traversal of the graph.

In Section 5 we report on extensive experiments showing that $\mathrm{PReaCH}$ performs very well.

\section{Related Work}

There has been intensive previous work on reachability indices. One interesting categorization [16] distinguishes between compression of the transitive closure [15, 5, 31, 17, 30], hop 
labeling, and refined online search. The former method gives very good query times for small instances yet does not scale to the larger graphs we are interested in here. We therefore do not consider it more closely. Hop-labeling assembles paths from labels stored with the nodes. In the most simple case of 2-hop labeling [9, 8, 7, 26, 6, 32, 18, each node stores a forward and backward set of nodes such that there is an $s$ - $t$ path if and only if the forward set of $s$ has a nonempty intersection with the backward set from $t$. There is also a 3-hop labeling technique where an additional lookup table for a small core set of nodes is used [20]. Here we compare our method with the two most recent 2-hop labeling methods which outperform previous methods based on hop-labeling and transitive closure compression: TF [6] uses topological folding to contract the graph in such a way that the maximal path length is halved in each contraction. PPL [32] uses pruned path labeling which can be viewed as a mix between 2-hop and 3-hop labeling since it does not store node sets explicitly but only entry points into paths.

Our technique PReaCH fits into the category refined online search [4, 28, 33, 34]. Here the idea is to precompute information stored with nodes and edges that allows to prune the search. GRAIL [34] is the most successful previous technique in this class which we therefore use as a basis for PReaCH.

Reachability indices and speedup techniques for computing shortest paths (e.g., in road networks) have striking similarities. For example, the 2-hop labeling technique [9] was immediately developed for both applications. In contrast, the 3-hop labeling technique [20] was developed independently from the analogous technique for shortest paths known as transit node routing [3]. The hierarchy based 2-hop labeling method described in [18] cites CHs and other hierarchical route planning methods as similar (and probably motivating) approaches. We see a particular resemblance to highway node routing [27]. However, [18] dismisses CHs as an approach to reachability because they conjecture that an excessive amount of shortcuts would be necessary. Hence, our RCHs without shortcuts can be viewed as a surprising result.

We demonstrate how the successful speedup technique Contraction Hierarchies $(\mathrm{CH})$ [12] can be adapted to the reachability problem. To demonstrate the analogy we outline the concept for shortest paths here and develop it for reachability in Section 3.1. CHs are built by successively removing (contracting) "unimportant" nodes from the graph. CHs make sure that shortest paths in the remaining graph are preserved. This is achieved by possibly inserting shortcuts. A query can then be performed by running a bidirectional variant of Dijkstra's algorithm on the full node set: the forward (backward) search only considers edges and shortcuts to (from) "more important" nodes. The construction ensures that some shortest path will be found this way.

\section{Preliminaries}

Consider a directed graph $G=(V, E)$. Let $n=|V|$ and $m=|E|$. A reachability query $(s, t)$ asks whether there is a path from $s$ to $t$ - in symbols $s \rightarrow t$. Queries with result true/false are called positive/negative respectively. The reachability problem can be reduced to the case of directed acyclic graphs (DAGs) as follows: Recall, that strongly connected components 
(SCCs) of a graph can be computed in linear time (e.g., [10]). One SCC is defined as a maximal set of nodes which are mutually reachable from each other. Hence, it suffices to check whether there is some path from the component of $s$ to the component of $t$ - this is equivalent to a reachability query in the acyclic component graph $G_{c}=\left(V_{c}, E_{c}\right)$. The SCCs are the nodes of the component graph and $E_{c}=\left\{(U, W) \in V_{c}^{2}:(U \times V) \cap E \neq \emptyset\right\}$. From now on we will therefore assume that $G$ is a DAG.

Breadth first search (BFS) from a node $s$ explores all nodes reachable from $s$ by visiting them in order of increasing shortest path distance. Since the edges are unweighted, this can be implemented by keeping unexplored nodes in a FIFO-queue. The reachability problem can be solved by running BFS: When $t$ is found, the BFS is aborted and true is returned. Otherwise, false is returned once all nodes reachable from $s$ are exhausted.

Reachability can also be tested using bidirectional BFS where BFS steps from $s$ alternate with BFS steps from $t$ on the backward graph $\bar{G}=(V, \bar{E})$ with $(u, v) \in \bar{E}$ whenever $(v, u) \in E$. When any node is reached from both sides, true is returned. When either search space is exhausted, false is returned. In the worst case, bidirectional BFS does twice the amount of work as unidirectional BFS and we also have considerable space overhead because we have to store both outgoing edges and incoming edges. However, in many positive queries and in negative queries where the backward search space is much smaller than the forward search space, we can be dramatically faster than unidirectional BFS.

Depth first search (DFS) explores the nodes of a graph in a recursive fashion: There is an outer loop through the nodes looking for roots $r$ for an exploration of the unexplored nodes reachable from $r$. The recursive function explore $(u)$ inspects the outgoing edges $(u, v)$ and recursively calls itself when $v$ has not been explored yet. DFS defines a spanning forest of the graph - one tree for each considered root. Let $\phi(v) \in 1$..n define the order in which the nodes are explored by DFS.1 Note that $\phi$ is a preordering of the nodes in each tree of the DFS forest. In particular, the nodes in a subtree $T$ of the forest rooted at $v$ have numbers starting at $\phi(v)$ and ending at $\phi(v)+|T|-1$.

A third useful way to explore the nodes of a DAG are topological levels [34]. Sources of the graph, i.e., nodes $v$ with out-degree zero have level $L(v)=0$. The remaining nodes have level $L(v)=1+\max \{L(u):(u, v) \in E\}$. Similarly, we can define backward topological levels based on sink nodes of the graph, i.e., nodes with in-degree zero.

\section{The PReaCH Reachability Index}

\subsection{Reachability Contraction Hierarchies}

In general, a Reachability Contraction hierarchy (RCH) can be defined based on any numbering order : $V \rightarrow 1$..n. We successively contract nodes with increasing order $(v)$. Contracting node $v$ means removing it from the graph and possibly inserting new edges such that the reachability relation in the new graph is the same as in the previous graph, i.e., if the graph contains a path $\langle u, v, w\rangle$ and after removing $v, u$ would no longer be reachable from $v$, we

\footnotetext{
${ }^{1}$ Throughout this paper $i . . j$ is a shorthand for $\{i, \ldots, j\}$.
} 
have to insert a new shortcut edge $(u, w)$. The query algorithm does not use the contracted versions of the graph but only the ordering and the shortcuts. This algorithm is based on the observation that in an RCH it suffices to look at "up-down" paths:

Theorem 1 In a graph $G, s \rightarrow t$ if and only if in an $R C H G^{\prime}$ obtained from $G$ there is a path of the form

$$
\left\langle s=u_{1}, \ldots, u_{k}=v=w_{1}, \ldots, w_{\ell}=t\right\rangle
$$

such that

$$
\begin{gathered}
\operatorname{order}\left(u_{1}\right)<\cdots<\operatorname{order}\left(u_{k}\right) \text { and } \\
\operatorname{order}\left(w_{1}\right)>\cdots>\operatorname{order}\left(w_{\ell}\right) .
\end{gathered}
$$

Proof: If there is an $s$ - $t$ path in $G^{\prime}$ then there must also be a path in $G$ since shortcuts never introduce new connections.

For the opposite direction, consider any $s$ - $t$ path $P$ in $G$. $P$ may not be an up-down path. However, we will show that it can be gradually transformed into an up-down path in $G^{\prime}$. Consider the subsequence $M:=\langle u, v, w\rangle$ of $P$ with $\operatorname{order}(v)<\operatorname{order}(u)$ and $\operatorname{order}(v)<$ $\operatorname{order}(w)$ which minimizes $\operatorname{order}(v)$, i.e., we consider the "lowest local minimum". When $v$ was contracted, two things may have happened. Either, there was another path $M^{\prime}$ from $v$ to $w$ in the graph at the time of contraction. Note that all nodes $u \in M^{\prime}$ have $\operatorname{order} \operatorname{order}(u)>\operatorname{order}(v)$. In this case, we replace $M$ by $M^{\prime}$ in $P$. Otherwise, the $\mathrm{RCH}$ construction inserted a shortcut edge $e=(u, w)$ into the graph. In this case, we replace $M$ by $e$ in $P$. Either way, we end up with a path from $s$ to $t$ whose lowest local minimum (if any) is higher than before. We can continue this process until no local minimum remains $P$ has been transformed into an up-down path.

Hence, a query can be implemented by a variant of the bidirectional BFS from Section 2 where both searches only look at adjacent nodes with larger order-value than the current node. A further modification is that now both search spaces must be exhausted in order to safely return false.

We now exploit the fact that any DAG contains source nodes (with in-degree zero) and sink nodes (with out-degree zero). Contracting such a node $v$ never requires the insertion of shortcuts because shortcuts always bridge paths of the form $\langle u, v, w\rangle$ but such paths cannot exist because $v$ either lacks incoming or outcoming edges. Hence from now on we restrict the considered orderings such that they only contract source or sink nodes $2^{2}$ This implies a huge simplification and acceleration of preprocessing compared to general RCHs. In particular, it suffices to use a static graph data structure and we get linear time preprocessing except perhaps for deciding which source or sink nodes should be contracted next. In contrast, a general $\mathrm{CH}$ might have to insert a quadratic number of shortcuts and indeed this is a significant limitation for computing shortest path $\mathrm{CHs}$ for graphs with a less pronounced hierarchy than road networks. In other words, RCHs have a much wider applicability and robustness than shortest path CHs.

\footnotetext{
${ }^{2}$ We also made experiments with more general RCHs. While this indeed improved query time by some small fraction, the overhead in terms of preprocessing time and space was considerable so that we did not explore this option any further.
} 


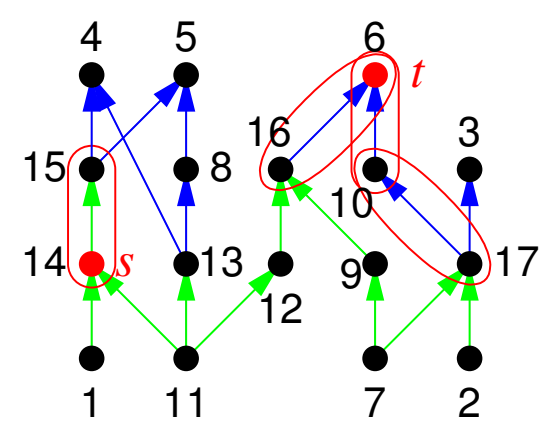

Figure 1: Example RCH. Edges in the forward search space are light green and those in the backward search space are dark blue. The search spaces for a query from $s$ to $t$ are circled. Node labels specify the node ordering.

There are still many ways to define an $\mathrm{RCH}$ ordering. We use the total degree (in-degree plus out-degree) of the nodes in the input graph for deciding in which order to contract source and sink nodes, i.e., nodes with smallest degree are contracted first. The ordering is computed on-line - a priority queue holds the source and sink nodes of the current graph using their degree in the input graph as priority. In each iteration, a lowest priority node $v$ is contracted. The idea behind our priority function is to delay contraction of high degree nodes and thus to limit the branching factor of the resulting query search spaces. Since no shortcuts are needed, the contraction process degenerates to a kind of graph traversal - a contracted node is not really removed but just marked as contracted and the degrees of its neighbors are decremented. Those nodes which become sources or sinks are inserted into the priority queue. Note that only $2 n$ priority queue operations are performed. In particular, no decrease key operations such as in Dijkstra's shortest path algorithm are needed. The running time of this algorithm is "near linear" in several senses. Using a comparison based priority queue, the running time becomes $\mathcal{O}(m+n \log n)$ with a quite small constant in front of the $n \log n$ term. In theory, we could use faster integer priority queues, for example van-Emde Boas trees [29, 23] which would yield running time $\mathcal{O}(m+n \log \log n)$.

Considering the RCH query algorithm, we can partition the edge set into two disjoint sets: edges $(u, v)$ with $\operatorname{order}(u)<\operatorname{order}(v)$ will only be considered by the forward search and the remaining ones only in the backward search. We organize the graph data structure in such a way that forward and backward search can directly access the edges they need. This implies that each edge is stored only once while ordinary bidirectional search requires us to store each edge twice. Hence, RCHs save a considerable amount of space.

Figure 1 gives an example graph marking the forward and backward edges and the search spaces for an example $s$ - $t$ query.

\subsection{Pruning Based on Topological Levels}

The central observation in [34] on topological levels is very simple: 


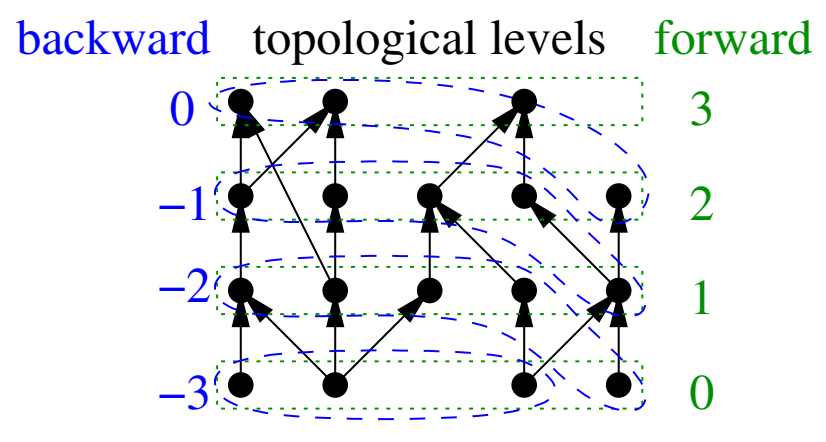

Figure 2: Forward and backward topological levels for an example graph.

Lemma $2 \forall u \neq v \in V: L(u) \geq L(v) \Rightarrow u \not \rightarrow v$.

We can apply Lemma 2 to $v$ and $t$ whenever we consider to explore a node $v$ in the forward search. Analogously, we can apply it to $s$ and $v$ whenever we consider a node $v$ in the backward search. Furthermore, we can apply the same reasoning to backward topological levels.

Figure 2 gives an example for forward and backward topological levels for the same graph as in Figure 1.

There are several ways to compute topological levels. We use a modified DFS. Each node $v$ maintains a counter $c(v)$ of its unexplored incoming edges (initialized to its in-degree) and a lower bound on its level $L(v)$ (initialized to 0 ). We perform a modified DFS using the sources of $G$ as root nodes. When an edge $(u, v)$ is considered, we update its level to $\max (L(v), 1+L(u))$ and decrement $c(v)$. Only if $c(v)$ dropped to zero, we recurse on $v$. We also keep track of the number of nodes reached from each source $r$ and store it as treeSize(r).

We could also apply a test like Lemma 2 to any topological ordering. However, note that we profit from the fact that Lemma 2 also excludes paths between different nodes in the same level and levels may contain many nodes. Hence, topological levels are stronger than topological orderings.

\subsection{Pruning Based on DFS Numbering}

Consider a numbering $f \rightarrow 1$..n of the nodes. One fairly general idea is to exploit the properties of $f$ in order to store a compressed, approximate representation of the set of nodes reachable from each node. We aim for a rather rough approximation that can be computed in linear time and space for the entire graph and where we can test in constant time whether a node is in this approximated set. By applying this test everywhere during a query, we can nevertheless obtain a significant amount of improvement. More concretely, we will store a constant number of ranges of node numbers that are either empty or full. When the destination node $t$ is in an empty range, the search does not have to continue there. When $t$ is in a full range, the entire search can be stopped with a positive result. Note the asymmetry between these two cases. For positive queries, a positive test result has a much 


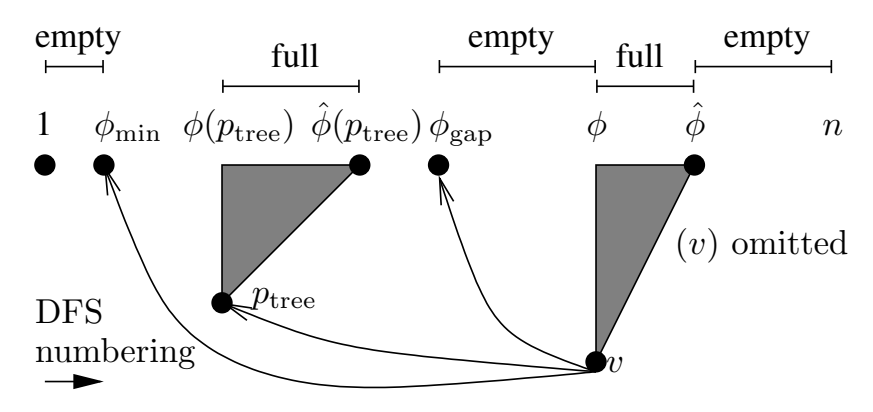

Figure 3: Full and empty intervals derived from a DFS ordering $\phi$. Shaded triangles indicate subtrees of the DFS tree.

bigger potential for improvement. As for topological levels, we only describe the case for forward search. For backward search, the same reasoning is applied on the backward graph.

The original version of GRAIL [33] is a special case of the above approach storing a single range (with respect to finishing time of the DFS) that must contain the target node. In a later version GRAIL 34] adds a positive range based on DFS numbering. GRAIL achieves additional pruning by working with several DFS searches. We take a different approach and extract more information from a single DFS numbering, obviating the need to compute and store finishing times, and still getting more useful information from a single DFS 3

Let $\phi(v)$ denote the DFS number of node $v$ and $\hat{\phi}(v)$ the largest DFS number of a node in the subtree of the DFS tree rooted at $v$. The properties of DFS ensure that the nodes in range $(v):=\phi(v) . . \hat{\phi}(v)$ are all reachable from $v$ (they form the subtree of the DFS tree which is rooted at $v$ ) and that no nodes with DFS number exceeding $\hat{\phi}(v)$ is reachable from $v$. Only this property of DFS numbering is already used in GRAIL [34]:

Lemma $3 \forall v, t \in V: \phi(t) \in \operatorname{range}(v) \Rightarrow v \rightarrow t$.

However, we also immediately get the following negative range:

Lemma $4 \forall v, t \in V: \phi(t)>\hat{\phi}(v) \Rightarrow v \not \rightarrow t$.

Proof: Assume to the contrary that for any node $v$ there is a node $t$ such that $v \rightarrow t$ yet $\phi(t)>\hat{\phi}(v)$. Consider a path $P$ from $v$ to $t$ and the first edge $(u, w)$ on $P$ with $\phi(w)>\hat{\phi}(v)$. This implies that $w$ is still unexplored when DFS finishes exploring $v$. In particular, when DFS explored $u, w$ was not explored yet. Hence, DFS should then have explored $w$ recursively - a contradiction.

Indeed, for any node $w$ with $v \rightarrow w$, range $(w)$ yields a range that we can use for positive pruning. We propose to actually compute and store the node outside range $(v)$ with the largest such range (or $\perp$ if no such node exists). It turns out this can be done while computing the DFS numbering.

\footnotetext{
${ }^{3}$ Of course nobody hinders us to get even better pruning from several DFS searches. However, we conjecture that the information in the finishing times is indeed redundant when using the DFS numbers in the way we do.
} 
Lemma 5 For any $v \in V$, consider the node $w=p_{\text {tree }}(v)$ with $v \rightarrow w$ and $w \notin \operatorname{range}(v)$ which maximizes $|\operatorname{range}(w)|$. When DFS on $v$ finishes, $w$ can be computed as

$$
\begin{gathered}
w:=\operatorname{maxind}\left\{\left|\operatorname{range}\left(p_{\text {tree }}(u)\right)\right|:(v, u) \in E \wedge p_{\text {tree }}(u) \neq \perp\right\} \cup \\
\{|\operatorname{range}(u)|:(v, u) \in E \wedge \phi(u)<\phi(v)\} .
\end{gathered}
$$

Proof: (Outline) The above equation follows easily by induction on the structure of the DAG, starting with the sinks of $G$. Moreover, all required values are known when DFS on $v$ finishes.

Similarly, we can exploit information available during the DFS to infer information that yields the empty interval to the left of any node reachable from $v$.

Lemma 6 For any $v \in V$, let $\phi_{\min }(v)$ denote the smallest DFS number of a node reachable from $v$. When DFS on $v$ finishes, $\phi_{\min }(v)$ can be computed as

$$
\phi_{\min }(v):=\min \left\{\phi_{\min }(w):(v, w) \in E\right\} \cup\{\phi(v)\}
$$

The proof is analogous to the proof of Lemma 5 .

Finally, we compute the following empty range just to the left of $v$;

Lemma 7 When DFS finishes for $v$, define

$$
\begin{aligned}
\phi_{\text {gap }}(v):=\max & \{\hat{\phi}(w):(v, w) \in E \wedge \phi(w)<\phi(v)\} \cup \\
& \left\{\phi_{\text {gap }}(w):(v, w) \in E\right\} .
\end{aligned}
$$

Then $\phi_{\text {gap }}(v)+1 . . \phi(v)$ is an empty range.

Proof: (Outline) Similar to the proof of Lemma 5 the definition of $\phi_{\text {gap }}(v)$ ensures that there can be no node between $\phi_{\text {gap }}(v)$ and $\phi(v)$ reachable from $v$-otherwise, $\phi_{\text {gap }}(v)$ would attain a larger value.

Figure 3 summarizes lemmas 2,7 .

There are many ways to define a DFS ordering: We are free to choose the order in which we scan the nodes for starting recursive exploration and we can choose the order in which we inspect edges leaving a node being explored. Indeed, we could compute several DFS orderings and use all of them for pruning searches. Our current implementation uses only a single ordering thus minimizing preprocessing time and space. We do not have very strong heuristics for finding good orderings but there is one heuristics that seems to be useful: Make sure that most nodes are in a small number of trees because this leads to large positive intervals. It therefore makes sense to only uses sources of the graph as tree roots. In addition, we order the sources by the number of nodes reached from them during the search for topological levels described in Section 3.2 . 


\section{Implementation Details}

We have implemented PReaCH using $\mathrm{C}++$. For the priority queues in $\mathrm{CH}$-ordering, we use the cache efficient implementation from [25]. All nodes, edges, and orderings are encoded using 32-bit integers. For each edge we store its destination node. For each node $v$ we store the following information: the first edge for the forward search space, $L(v)$, $\phi(v), \hat{\phi}(v), \phi_{\min }(v), \phi_{\text {gap }}(v), \phi\left(p_{\text {tree }}(v)\right)$, and $\hat{\phi}\left(p_{\text {tree }}(v)\right)$. For all these node-values, we need separate information for forward and backward search. Note that there is no need to store the order in the $\mathrm{CH}$ since the separation into forward and backward edges is all we need. All these integers are stored consecutively in memory so that they can be accessed quite cache efficiently. Note that the obvious space saving measure of storing only $p_{\text {tree }}(v)$ rather than both $\phi\left(p_{\text {tree }}(v)\right)$, and $\hat{\phi}\left(p_{\text {tree }}(v)\right)$ would incur additional cache faults during the query. The overall resulting space complexity of the index data structure is $4 m+64 n$ bytes.

During a query, the pruning rules resulting from Lemmas 2-7] are applied aggressively, i.e., for both forward and backward search, and even before a node is queued. Both forward and backward topological levels are used.

\section{Experiments}

All experiments have been performed using a single core of an Intel Xeon X5550 running at $2.67 \mathrm{GHz}$ with $8 \mathrm{MB}$ Level3 cache, $256 \mathrm{kB}$ Level 2 cache and $48 \mathrm{~GB}$ of DDR3 RAM. The system ran Ubuntu 12.04.2 using a Linux kernel 3.5. The code has been compiled using gcc 4.8.2 with optimization level 03.

As far as sensible, we adopt instances and measurement conventions from previous work to improve comparability. In table, best values are bold. $\mathrm{K}$ and $\mathrm{M}$ are shorthands for 000 and 000000 respectively.

\subsection{Instances}

We use graphs of five categories, largely adopted from [34, 17, 6]. Table 1 summarizes their properties. In addition we have added Kronecker graphs as a family of graphs that have become a standard in benchmarking graph algorithms and can be generated with arbitrary size. Besides the number of nodes and edges we give a number of further important parameters. We see that the edge density $m / n$ is very small (even close to one) for many instances. We will see that the graphs with larger $m / n$ can be much more difficult to handle. Another column gives the length $d$ of the longest path which turns out to be fairly small for all instances except the Kronecker graphs. Finally, we indicate the fraction of positive queries in a random sample of 100000 queries. It turns out that this fraction is close to zero for most instances. Since an application is not guaranteed to have the same small rate of positive queries, we explicitly use specially generated positive query instances in our experiments. 
Table 1: Instances used for our experiments. $d$ is the maximal path length.

\begin{tabular}{|c|c|c|c|c|c|}
\hline Dataset & Nodes & Edges & $m / n$ & $d$ & \%pos \\
\hline \multicolumn{6}{|l|}{ Kronecker } \\
\hline kron12 & $2^{12}$ & $117 \mathrm{~K}$ & 28.60 & 279 & 28 \\
\hline kron17 & $2^{17}$ & $5069 \mathrm{~K}$ & 38.68 & 1354 & 19 \\
\hline kron22 & $2^{22}$ & $184 \mathrm{M}$ & 43.95 & 5821 & 13 \\
\hline \multicolumn{6}{|l|}{ large random } \\
\hline rand100m5x & $100 \mathrm{M}$ & $500 \mathrm{M}$ & 5 & 37 & 0.0 \\
\hline rand $100 \mathrm{~m} 2 \mathrm{x}$ & $100 \mathrm{M}$ & $200 \mathrm{M}$ & 2 & 21 & 0.0 \\
\hline rand10m10x & $10 \mathrm{M}$ & $100 \mathrm{M}$ & 10 & 60 & 5.0 \\
\hline $\operatorname{rand} 10 \mathrm{~m} 5 \mathrm{x}$ & $10 \mathrm{M}$ & $50 \mathrm{M}$ & 5 & 35 & 0.0 \\
\hline $\operatorname{rand} 10 \mathrm{~m} 2 \mathrm{x}$ & $10 \mathrm{M}$ & $20 \mathrm{M}$ & 2 & 19 & 0.0 \\
\hline rand1m10x & $1 \mathrm{M}$ & $10 \mathrm{M}$ & 10 & 59 & 10 \\
\hline rand $1 \mathrm{~m} 5 \mathrm{x}$ & $1 \mathrm{M}$ & $5 \mathrm{M}$ & 5 & 33 & 0.2 \\
\hline $\operatorname{rand} 1 \mathrm{~m} 2 \mathrm{x}$ & $1 \mathrm{M}$ & $2 \mathrm{M}$ & 2 & 19 & 0.0 \\
\hline \multicolumn{6}{|l|}{ large real } \\
\hline citeseer & $694 \mathrm{~K}$ & $312 \mathrm{~K}$ & 0.45 & 13 & 0.0 \\
\hline citeseerx & $6540 \mathrm{~K}$ & $15 \mathrm{M}$ & 2.30 & 59 & 0.2 \\
\hline cit-patents & $3775 \mathrm{~K}$ & $17 \mathrm{M}$ & 4.38 & 32 & 0.1 \\
\hline go-uniprot & $6968 \mathrm{~K}$ & $35 \mathrm{M}$ & 4.99 & 21 & 0.0 \\
\hline uniprot $22 \mathrm{~m}$ & $1595 \mathrm{~K}$ & $1595 \mathrm{~K}$ & 1.00 & 4 & 0.0 \\
\hline uniprot100m & $16 \mathrm{M}$ & $16 \mathrm{M}$ & 1.00 & 9 & 0.0 \\
\hline uniprot $150 \mathrm{~m}$ & $25 \mathrm{M}$ & $25 \mathrm{M}$ & 1.00 & 10 & 0.0 \\
\hline \multicolumn{6}{|l|}{ small real dense } \\
\hline arxiv & 6000 & $67 \mathrm{~K}$ & 11.12 & 167 & 15 \\
\hline citeseer-sub & $11 \mathrm{~K}$ & $44 \mathrm{~K}$ & 4.13 & 36 & 0.4 \\
\hline go & 6793 & $13 \mathrm{~K}$ & 1.97 & 16 & 0.2 \\
\hline pubmed & 9000 & $40 \mathrm{~K}$ & 4.45 & 19 & 0.7 \\
\hline yago & 6642 & $42 \mathrm{~K}$ & 6.38 & 13 & 0.2 \\
\hline \multicolumn{6}{|l|}{ small real sparse } \\
\hline agrocyc & $13 \mathrm{~K}$ & $14 \mathrm{~K}$ & 1.07 & 16 & 0.1 \\
\hline amaz & 3710 & 3947 & 1.06 & 16 & 17 \\
\hline anthra & $12 \mathrm{~K}$ & $13 \mathrm{~K}$ & 1.07 & 16 & 0.1 \\
\hline ecoo & $13 \mathrm{~K}$ & $14 \mathrm{~K}$ & 1.08 & 22 & 0.1 \\
\hline human & $39 \mathrm{~K}$ & $40 \mathrm{~K}$ & 1.01 & 18 & 0.0 \\
\hline kegg & 3617 & 4395 & 1.22 & 26 & 20 \\
\hline mtbrv & 9602 & $10 \mathrm{~K}$ & 1.09 & 22 & 0.2 \\
\hline nasa & 5605 & 6538 & 1.17 & 35 & 0.6 \\
\hline vchocyc & 9491 & $10 \mathrm{~K}$ & 1.09 & 21 & 0.1 \\
\hline xmark & 6080 & 7051 & 1.16 & 38 & 1.4 \\
\hline \multicolumn{6}{|l|}{ stanford } \\
\hline email-EuAll & $231 \mathrm{~K}$ & $223 \mathrm{~K}$ & 0.97 & 7 & 5 \\
\hline -Gnutella31 & $48 \mathrm{~K}$ & $55 \mathrm{~K}$ & 1.15 & 14 & 0.8 \\
\hline soc-LiveJournal1 & $971 \mathrm{~K}$ & $1024 \mathrm{~K}$ & 1.05 & 24 & 21 \\
\hline web-Google & $372 \mathrm{~K}$ & $518 \mathrm{~K}$ & 1.39 & 34 & 15 \\
\hline wiki-Talk & $2282 \mathrm{~K}$ & $2312 \mathrm{~K}$ & 1.01 & 8 & 0.8 \\
\hline
\end{tabular}


Kronecker: We interpret the graphs generated by the RMAT generator used in the Graph500 benchmark [24] as DAGs, i.e., interpreting an edge $\{u, v\}$ as the directed edge $(\min (u, v), \max (u, v))$. The name $\operatorname{kron}\{x\}$ stands for a Kronecker graph with $2^{x}$ nodes generated using the default parameters from the Graph500 benchmark.

Large Random: These graphs are randomly generated DAGs. They are graphs with given number of nodes and $m$ edge pairs chosen independently at random [34]. We use the naming scheme rand $\{n\}\{D\} \mathrm{x}$, where $n$ is the number of nodes (e.g.: $1 \mathrm{~m}$ for 1 million) and $D=m / n$. We also look at larger instances from this family than in previous works.

Large Real: The authors of GRAIL [34] introduced seven larger graphs to demonstrate their scaling abilities. citeseer, citeseerx and cit-patents are citation networks, go-uniprot is a joint graph of Gene Ontology terms and the annotations file from the UniProt database. The other graphs (uniprot22m, uniprot $100 \mathrm{~m}$, uniprot $150 \mathrm{~m}$ ) are subsets of the UniProt RDF graph.

Small Real Dense: These graphs with less than 67000 edges are mostly obtained from citation networks (pubmed, citeseer, arxiv) [19]. They have larger $m / n$ which makes them more difficult to handle for reachability indices.

Small Real Sparse: These graphs have an average degree less than 1.22 and less then 40000 nodes. xmark and nase represent XML documents; amaze and kegg are metabolic networks. The others are from from BioCyc [34]. They represent pathway and genome databases.

Stanford: These graphs from the Stanford Large Network Dataset Collection were initially used for evaluating TF [6]. Thy represent an email network from an EU research institution (email-EuAl1), the Gnutella peer to peer network from August 31 2002 (p2p-Gnutella31), the LiveJournal online social network (soc-LiveJournal1), a (contracted) web graph from Google (web-Google) and the Wikipedia communication network (wiki-Talk).

Most experiments average times for $100000 s$-t reachability queries. We use three different types:

random: Random queries provided by randomly picking $s, t \in V(G), s \neq t$

positive: Positive queries obtained by picking a nonisolated $s \in V$ uniformly at random and then picking a node reachable from $s$ uniformly at random.

negative: Negative queries obtained by randomly picking $s \in V$ uniformly at random and then picking a node not reachable from $s$ uniformly at random.

We distinguish between positive and negative queries since these can behave very differently, in particular some instances have very few positive queries among random queries so that only measuring random queries almost ignore positive queries which may be very important in real applications. 
Table 2: Average query time of bidirectional BFS for positive and negative queries compared to PReaCH and its constituent heuristics.

\begin{tabular}{|c|c|c|c|c|c|c|c|c|c|c|}
\hline & \multicolumn{2}{|c|}{$\begin{array}{l}\text { bidir. BFS } \\
\text { avg. query time in ns }\end{array}$} & \multicolumn{2}{|c|}{$\begin{array}{l}\text { RCH } \\
\text { speedup vs BFS }\end{array}$} & \multicolumn{2}{|c|}{$\begin{array}{c}\text { Levels } \\
\text { speedup vs BFS }\end{array}$} & \multicolumn{2}{|c|}{$\begin{array}{l}\text { DFS-Trees } \\
\text { speedup vs BFS }\end{array}$} & \multicolumn{2}{|c|}{$\begin{array}{c}\text { PReaCH } \\
\text { speedup vs BFS }\end{array}$} \\
\hline & + & - & + & - & + & - & + & - & + & - \\
\hline kron12 & $31 \mathrm{~K}$ & $42 \mathrm{~K}$ & 3.20 & 1.56 & 1.61 & 2688 & 282 & 53.26 & 668 & 3440 \\
\hline kron17 & $2483 \mathrm{~K}$ & $2286 \mathrm{~K}$ & 4.91 & 1.21 & 2.03 & $56 \mathrm{~K}$ & 638 & 1490 & 4723 & $72 \mathrm{~K}$ \\
\hline kron22 & $130 \mathrm{M}$ & $198 \mathrm{M}$ & 4.00 & 2.17 & 1.06 & $864 \mathrm{~K}$ & 780 & 6544 & $97 \mathrm{~K}$ & $2330 \mathrm{~K}$ \\
\hline 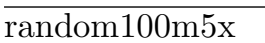 & $940 \mathrm{~K}$ & $748 \mathrm{~K}$ & 20.44 & 25.41 & 2.93 & 11.73 & 5.53 & 14.72 & 29.48 & 61.60 \\
\hline random $100 \mathrm{~m} 2 \mathrm{x}$ & 6475 & 8166 & 1.44 & 1.49 & 1.12 & 2.92 & 2.21 & 3.09 & 2.84 & 5.71 \\
\hline random10m10x & $159 \mathrm{M}$ & $91 \mathrm{M}$ & 131 & 143 & 5.58 & 46.72 & 7.66 & 30.63 & 203 & 638 \\
\hline random $10 \mathrm{~m} 5 \mathrm{x}$ & $415 \mathrm{~K}$ & $301 \mathrm{~K}$ & 25.34 & 30.65 & 2.66 & 12.89 & 4.78 & 13.04 & 29.15 & 62.24 \\
\hline random $10 \mathrm{~m} 2 \mathrm{x}$ & 2156 & 2318 & 1.78 & 1.88 & 1.22 & 3.25 & 2.63 & 3.19 & 3.03 & 3.96 \\
\hline random $1 \mathrm{~m} 10 \mathrm{x}$ & $18 \mathrm{M}$ & $11 \mathrm{M}$ & 43.90 & 32.98 & 5.78 & 54.48 & 8.79 & 33.37 & 91.69 & 277 \\
\hline random $1 \mathrm{~m} 5 \mathrm{x}$ & $284 \mathrm{~K}$ & $192 \mathrm{~K}$ & 24.30 & 28.03 & 2.70 & 12.80 & 4.83 & 12.83 & 28.97 & 53.94 \\
\hline random $1 \mathrm{~m} 2 \mathrm{x}$ & 1349 & 1420 & 2.02 & 2.05 & 1.25 & 4.82 & 3.63 & 4.55 & 4.48 & 6.67 \\
\hline cit-Patents & $381 \mathrm{~K}$ & $131 \mathrm{~K}$ & 21.90 & 17.33 & 1.72 & 18.83 & 2.70 & 14.80 & 26.69 & 86.99 \\
\hline citese & $101 \mathrm{~K}$ & 143 & 708 & 0.90 & 0.96 & 2.72 & 3042 & 1.67 & 2530 & 4.07 \\
\hline citeseerx & $3727 \mathrm{~K}$ & $1459 \mathrm{~K}$ & 2987 & 1075 & 1.19 & 4881 & 1418 & 3688 & 7611 & $11 K$ \\
\hline go-uniprot & $18 \mathrm{M}$ & 1812 & $28 \mathrm{~K}$ & 1.84 & 1.41 & 3.97 & 2.78 & 1.48 & $41 \mathrm{~K}$ & 33.10 \\
\hline uniprotenc- $22 \mathrm{~m}$ & $3661 \mathrm{~K}$ & 126 & $27 \mathrm{~K}$ & 0.52 & 1.35 & 6.14 & $128 \mathrm{~K}$ & 0.91 & $97 \mathrm{~K}$ & 3.85 \\
\hline uniprotenc- $100 \mathrm{~m}$ & $45 \mathrm{M}$ & 559 & $82 \mathrm{~K}$ & 0.74 & 1.57 & 6.23 & $1059 \mathrm{~K}$ & 1.07 & $851 \mathrm{~K}$ & 7.22 \\
\hline uniprotenc- $150 \mathrm{~m}$ & $71 \mathrm{M}$ & 849 & $90 \mathrm{~K}$ & 0.79 & 1.46 & 5.98 & $1446 \mathrm{~K}$ & 1.31 & $1193 \mathrm{~K}$ & 8.25 \\
\hline arxiv & $35 \mathrm{~K}$ & $30 \mathrm{~K}$ & 5.23 & 1.44 & 2.17 & 109 & 16.00 & 33.92 & 88.03 & 168 \\
\hline citeseer-sub & 2835 & 1236 & 9.89 & 2.50 & 1.47 & 7.13 & 4.97 & 6.07 & 22.70 & 20.47 \\
\hline go & 93.51 & 422 & 1.03 & 1.39 & 0.85 & 11.46 & 1.83 & 5.04 & 1.89 & 13.91 \\
\hline pubmed & 5076 & 1589 & 10.82 & 2.41 & 1.48 & 22.42 & 2.13 & 10.24 & 25.44 & 31.09 \\
\hline yago & 4968 & 185 & 67.52 & 1.02 & 0.88 & 2.25 & 26.07 & 2.87 & 112 & 9.64 \\
\hline agrocyc & 1173 & 225 & 17.06 & 3.89 & 1.03 & 39.57 & 114 & 7.55 & 90.88 & 31.05 \\
\hline ama & 2218 & 5070 & 53.78 & 121 & 1.21 & 609 & 229 & 165 & 179 & 597 \\
\hline thra & 879 & 206 & 13.19 & 3.67 & 0.97 & 37.19 & 83.03 & 6.89 & 66.72 & 29.74 \\
\hline & 1256 & 243 & 16.19 & 4.05 & 1.16 & 42.92 & 119 & 8.29 & 93.08 & 33.79 \\
\hline human & 1034 & 173 & 14.12 & 3.20 & 1.05 & 26.27 & 96.90 & 3.15 & 77.92 & 23.18 \\
\hline kegg & 2288 & 5991 & 46.78 & 128 & 1.34 & 492 & 222 & 191 & 169 & 668 \\
\hline mtbrv & 1083 & 242 & 14.36 & 4.11 & 1.08 & 41.52 & 107 & 7.71 & 82.63 & 33.61 \\
\hline & 255 & 540 & 3.20 & 4.67 & 1.14 & 26.24 & 4.22 & 10.77 & 11.08 & 25.47 \\
\hline vchocyc & 904 & 228 & 11.92 & 3.83 & 0.99 & 39.18 & 84.16 & 7.82 & 71.12 & 31.29 \\
\hline xmark & 734 & 1366 & 2.19 & 9.15 & 1.31 & 62.33 & 4.33 & 29.11 & 17.63 & 62.87 \\
\hline email-E & $250 \mathrm{~K}$ & $45 \mathrm{~K}$ & 2462 & 375 & 1.31 & 1482 & 8367 & 579 & 8267 & 1648 \\
\hline p2p-Gnute & 225 & 7133 & 4.21 & 116 & 0.88 & 843 & 7.25 & 191 & 6.49 & 710 \\
\hline -LiveJournal1 & $720 \mathrm{~K}$ & $1719 \mathrm{~K}$ & 3142 & 7908 & 1.35 & $34 \mathrm{~K}$ & $14 \mathrm{~K}$ & $11 \mathrm{~K}$ & $14 \mathrm{~K}$ & $56 \mathrm{~K}$ \\
\hline web-Google & $239 \mathrm{~K}$ & $542 \mathrm{~K}$ & 1351 & 2891 & 1.05 & $10 \mathrm{~K}$ & 4723 & 4709 & 4688 & $12 \mathrm{~K}$ \\
\hline wiki-Talk & $27 \mathrm{~K}$ & $283 \mathrm{~K}$ & 136 & 1159 & 1.08 & 8140 & 500 & 2031 & 371 & 8128 \\
\hline
\end{tabular}

\subsection{Contributions of the Individual Heuristics}

Table 2 compares bidirectional BFS with RCHs alone (Section 3.1), topological levels alone (Section 3.2), DFS numberings alone (Section 3.3), and PReaCH (the combination of all 
heuristics). We use BFS as a baseline since it seems to be more robust than unidirectional search which can take a long time even if the search space in one direction is very small. We see that PReaCH achieves average speedup between about two and two millions. There is never a slowdown and small single digit speedups only occur for easy instances where even BFS achieves query times in the microsecond range.

Each individual heuristics occasionally has speedup below one (i.e., a slight slowdown). However, this only happens for very easy instances. No individual heuristics achieves performance comparable to full PReaCH over all instances. For individual instances, PReaCH is sometimes slightly outperformed by topological levels for negative queries and by DFS-Trees for positive queries.

RCHs alone are quite good for positive queries but less effective for negative queries. This makes a combination with pruning heuristics essential to achieve overall good performance. Since the pruning heuristics work very well for negative queries, the two approaches complement each other.

Topological levels are good for negative queries but much less so for positive queries. This is not surprising since for many negative queries they can disprove reachability from the start by just comparing the levels of $s$ and $t$. DFS-Trees are very good at reducing the search space size of otherwise difficult positive queries.

\subsection{Comparison with other Approaches}

There are so many reachability indices that it is impossible to compare with all of them directly. We have therefore focused on three recent techniques that fare very well in comparison with others and seem to constitute the state of the art.

GRAIL [34] is particularly interesting since, similar to PReaCH, it has guaranteed linear preprocessing time and space. The authors recommend a variant using data from five DFS traversals which we call GRAIL5 and which we use in most comparisons.4 Incidentally, GRAIL5 also uses about the same amount of space than PReaCH so that this additionally simplifies the analysis. In some experiments we also look at the more light weight variant with a single DFS and call it GRAIL.

TF [6] is a more recent labelling technique based on "folding" paths. It is particularly, useful for graphs with small value of $d$ in Table 1. PPL [32] is the most recent technique which appeared only a few weeks before the submission of this paper. It is also a labelling technique and very often achieves quite small labels, excellent query time and good preprocessing time. In particular, it can profit from long paths in the graph.

Table 3 summarizes the results giving absolute values for PReaCH and slowdown factors relative to PReaCH for the other heuristics. PReaCH dominates GRAIL5 with respect to both query time and preprocessing time (while using about the same space). The advantage is particularly pronounced for positive queries where the improvement is often more than an order of magnitude. The significant advantage of PReaCH over GRAIL5 with respect to

\footnotetext{
${ }^{4}$ More precisely, we are using the settings -dim 5 -t -2 -ltype 1 in the code from April 2011 downloaded from http://code.google.com/p/grail/.
} 
Table 3: Performance of PReaCH compared to GRAIL [34 with five DFS numberings, TF [6], and PPL [32]. The numbers for PReaCH are average execution time in ns for queries an and total construction time in ms. The other numbers are slowdown (or space overhead for columns "ind") relative to PReaCH.

\begin{tabular}{|c|c|c|c|c|c|c|c|c|c|c|c|c|c|c|}
\hline & \multicolumn{3}{|c|}{ PReaCH } & \multicolumn{3}{|c|}{ GRAIL5 } & \multicolumn{4}{|c|}{$\mathrm{TF}$} & \multicolumn{4}{|c|}{ PPL } \\
\hline & + & - & constr & + & - & constr & + & 列 & constr & ind & + & - & constr & \\
\hline on12 & 45.88 & 11.86 & 7.30 & 54.71 & 7.20 & 4.18 & 3.88 & 20.64 & 4152 & 27.02 & 0.50 & 1.59 & 3.81 & 0.1 \\
\hline on17 & 524 & 31.24 & & 48.43 & 10.34 & 3.16 & - & - & - & - & 17 & 1.82 & 3.04 & 1 \\
\hline on22 & 1546 & 86.03 & $74 K$ & 459 & 6.66 & 1.99 & - & - & - & - & 13 & 1.56 & 2.33 & 0.1 \\
\hline ndor & 31K & $12 \mathrm{~K}$ & $622 \mathrm{~K}$ & - & - & - & - & - & - & - & - & - & - & \\
\hline & 2267 & 1422 & & 1.15 & 0.50 & 3.24 & 0.22 & .20 & 3.01 & 0.67 & 0.27 & 0.27 & 5.11 & 0.38 \\
\hline & $784 K$ & $184 \mathrm{~K}$ & $70 K$ & 25.54 & 18.49 & 2.04 & - & - & - & - & - & - & - & \\
\hline & $13 \mathrm{~K}$ & 4831 & $41 K$ & 5.58 & 7.67 & 2.34 & 0.06 & 0.12 & .78 & 1.81 & 0.12 & 0.14 & 2.16 & \\
\hline & 719 & 589 & $21 K$ & 2.66 & 1.13 & 3.00 & 0.40 & 0.30 & 3.38 & 0.67 & 0.48 & 0.35 & 4.11 & 0.3 \\
\hline & $204 \mathrm{~K}$ & $41 \mathrm{~K}$ & 5297 & 11.33 & 8.26 & 1.76 & - & - & - & - & 0.04 & 0.09 & 324 & 81. \\
\hline & 06 & 3158 & 3120 & 5.49 & 5.67 & 2.12 & 0.06 & 0.14 & .53 & 11.90 & 0.11 & .13 & 37.65 & \\
\hline & 301 & 213 & 1635 & 4.53 & 1.75 & 2.81 & 0.56 & 0.52 & 3.44 & 0.67 & 0.78 & 0.65 & 4.28 & 0.3 \\
\hline cit- $\mathrm{Pe}_{\mathrm{c}}$ & $14 \mathrm{~K}$ & 519 & 64 & 88 & .71 & 2.31 & 0.06 & 0.35 & 2.94 & 16.70 & 06 & .20 & 6.20 & 2.3 \\
\hline & 07 & 35.25 & 305 & 4.32 & 3.11 & 7.28 & 2.71 & 0.26 & 66 & 38 & .05 & 81 & .76 & \\
\hline & 488 & 131 & 7127 & 5.80 & 3.00 & 3.02 & 2.04 & 1.42 & 86 & 3.72 & .54 & 30 & 63 & 0.2 \\
\hline & 450 & 54.59 & 6097 & 3.41 & 1.93 & 5.78 & 2.50 & 0.91 & 10.37 & 0.38 & 56 & .71 & 30 & \\
\hline $\mathrm{m}$ & 6.03 & 32.55 & 402 & 22.25 & 2.76 & 3.38 & 1.79 & 1.15 & .53 & 0.41 & 15 & 62 & .89 & 0.1 \\
\hline & 3.88 & 78.47 & 6072 & 1.11 & 2.30 & 12.09 & .60 & 1.21 & 6.56 & 41 & .56 & .55 & .74 & 1 \\
\hline uniprot & 0.15 & 103 & $10 K$ & 22.32 & 2.22 & 12.03 & 3.00 & 1.12 & 5.52 & 0.41 & 3.40 & 2.16 & 4.53 & 0.1 \\
\hline$x$ & 408 & 181 & & 52 & .0 & 2.82 & 65 & 2.38 & 28 & 3.93 & 14 & .2 & .83 & .5 \\
\hline & 126 & 60.06 & 6 & 15 & $2 .($ & 3 & 54 & 66 & 91 & & 0.59 & $r$ & .27 & 0.3 \\
\hline & 49.85 & 30.59 & & 5.50 & 1.9 & 3.81 & 0.94 & 94 & & 7 & .21 & 1.22 & .67 & 0.4 \\
\hline & 201 & 51.24 & & 5.75 & 2.62 & 3.31 & 0.46 & 0.79 & & & 0.38 & 0.88 & 4.76 & 0. \\
\hline yago & 44.58 & 19.39 & 3.45 & 9.76 & 2.66 & 4.06 & 2.10 & 0.87 & 16.91 & 0.68 & 1.15 & 1.96 & 4.76 & 0. \\
\hline & 69 & 71 & 0 & 2 & 4.99 & 3 & 10 & 9 & 4 & 5 & 19 & 3.82 & 5 & 0.2 \\
\hline & & 11 & 5 & & 3.27 & & & 3 & & & & & 5 & 0.2 \\
\hline & & 6 & 2 & & 5.2 & 7. & 14 & 2.18 & & & 6 & & 24 & 0.2 \\
\hline & & 4 & & 3 & 5.44 & & & 2.26 & & & .00 & D & 11 & .2 \\
\hline & & 5 & & 90 & 8.47 & 1 & 16.81 & 1.93 & & & 18 & 4 & .81 & 0.2 \\
\hline & 17 & 8.95 & 0 . & 18 & 3.4 & 6 & 1.42 & 1.18 & 53 & & .08 & .54 & 14.24 & 0.2 \\
\hline & 64 & 7.24 & 1 & 11 & 4.92 & 7.27 & 1.37 & 1.90 & 77 & 7 & 2.14 & .74 & 8.01 & .2 \\
\hline & 01 & 21.07 & 1. & 30 & 2.27 & & 5 & 1.18 & & & .09 & .42 & 7.92 & . \\
\hline & 24 & 7.73 & 1.57 & 17.78 & 4.60 & ) & 2.56 & 2.04 & 45 & & 2.02 & 3.55 & 8.18 & .2 \\
\hline sma & .37 & 21.71 & 1.31 & 5.91 & 2.88 & 5.65 & 1.88 & 1.18 & 20.69 & 0.65 & 1.12 & 1.43 & 10.53 & 0.4 \\
\hline & & & & & 3.1 & & & & & & & & & 5.7 \\
\hline & & & & & & & & & & & & & & 0.2 \\
\hline & 39 & 30.02 & & & . & & & 1.29 & & & 2.73 & 2 & 99 & . \\
\hline & .81 & 42.06 & 2 & 21 & 3.8 & & 1.82 & 1.11 & .23 & & 2.29 & 1.88 & .09 & 0 。 \\
\hline N⿴囗十 & .53 & 34.85 & 925 & 17.13 & 2.66 & 8.91 & 2.56 & 1.18 & 1.79 & 0.41 & 2.44 & 3.49 & 4.36 & 0 \\
\hline
\end{tabular}




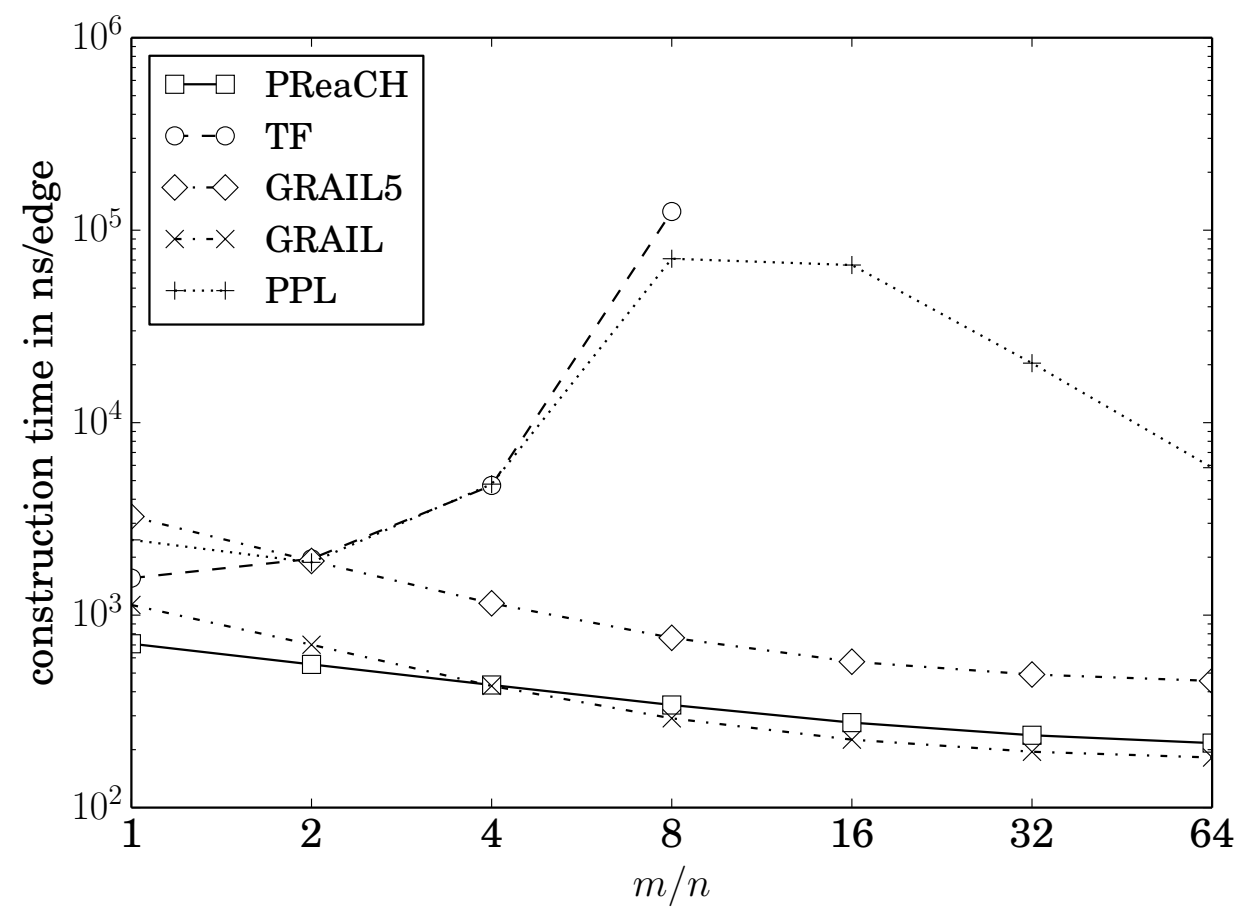

Figure 4: Construction time per edge for random DAGs with $n=100000, m / n=2 \ldots 64$

preprocessing is surprising since both technique traverse the graph five times (for PReaCH: RCHs, forward/backward topological levels, forward/backward DFS) and since PReaCH has additional overhead for a priority queue. The reason may be implementation details or deteriorating cache efficiency due to the randomization of DFS used in GRAIL. Neither TF nor PPL dominate GRAIL5 because they often need much higher preprocessing time.

Comparing PReaCH with TF and PPL is more complicated. With respect to construction time, PReaCH is always the best algorithm - sometimes by orders of magnitude. For the most difficult instances TF ran out of memory. For random10M10x PPL was stopped after 9h. With respect to query time, $\mathrm{PReaCH}$ achieves the best values for 43 out of 72 cases while TF ranks second with 16 best values closely followed by PPL with 13 best values. Basically, for easy instances, PReaCH slightly outperforms the labeling techniques. For difficult instances which the labeling techniques can handle at all, they significantly outperform PReaCH but at the cost of very high preprocessing time (and space in case of TF). With respect to space consumption, PPL is the best in almost all cases. However, for dense random graphs and for cit-Patents much more space is needed than for PReaCH, i.e., PReaCH can still score for being more predictable with respect to space consumption.

In order to get a feeling how the performance of the algorithms varies with the size and density of the graphs, we performed scaling experiments with random graphs. Figures 447 plot the results.

With respect to construction time, PReaCH and GRAIL show the expected near linear 


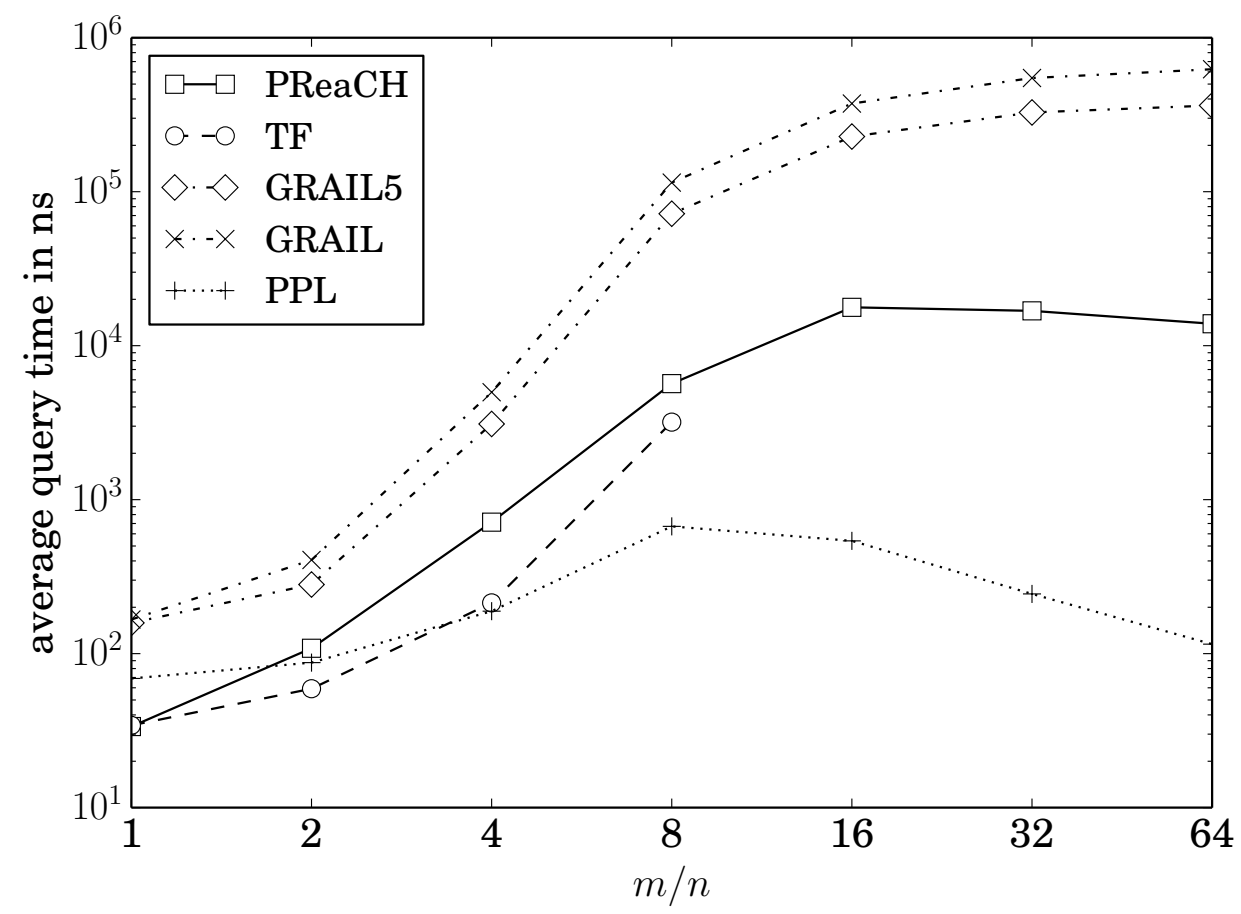

Figure 5: Average query time for random DAGs with $n=100000, m / n=2 \ldots 64$

behavior, i.e. near constant time per edge. Both curves slightly grow with input size probably due to cache effects. Both TF and PPL scale much worse with respect to graph size or density at least for small $m / n$. Interestingly, PPL first gets worse as $m / n$ goes up to 8 and then becomes better again. Probably for sufficiently dense random graphs, one can identify a small number of paths witnessing most existing connections and the path labelling of PPL can profit from this. For fixed $m / n$, PPL's construction time scales very badly with growing $n$. TF seems to gain ground on PPL with growing $n$ but it cannot cash in on this perspective since it runs out of memory for large $n$.

The query times of GRAIL and PReaCH first grow with growing density and then flatten. In particular, PReaCH profits once the edge density exceeds 16. For PPL, we see the same rise and fall as for the construction time when scaling $m / n$ with a maximum query time at $m / n=8$. Even at $m / n=8$, PPL has the best query times for random graphs outclassing all other algorithms for dense graphs.

Most previous studies concentrate on average query times. We believe that this can be misleading and thus also look at query time distributions now. Figures 8,10 show query time distributions for three large instances from three different categories. Data for more instances will be published with the technical report. The pruning based algorithms GRAIL and PReaCH show fluctuations of query time varying over several orders of magnitude. For example, for positive queries, instance wiki-Talk and GRAIL5 there are almost three orders of magnitude between the maximal query time and the median query time. PReaCH is 


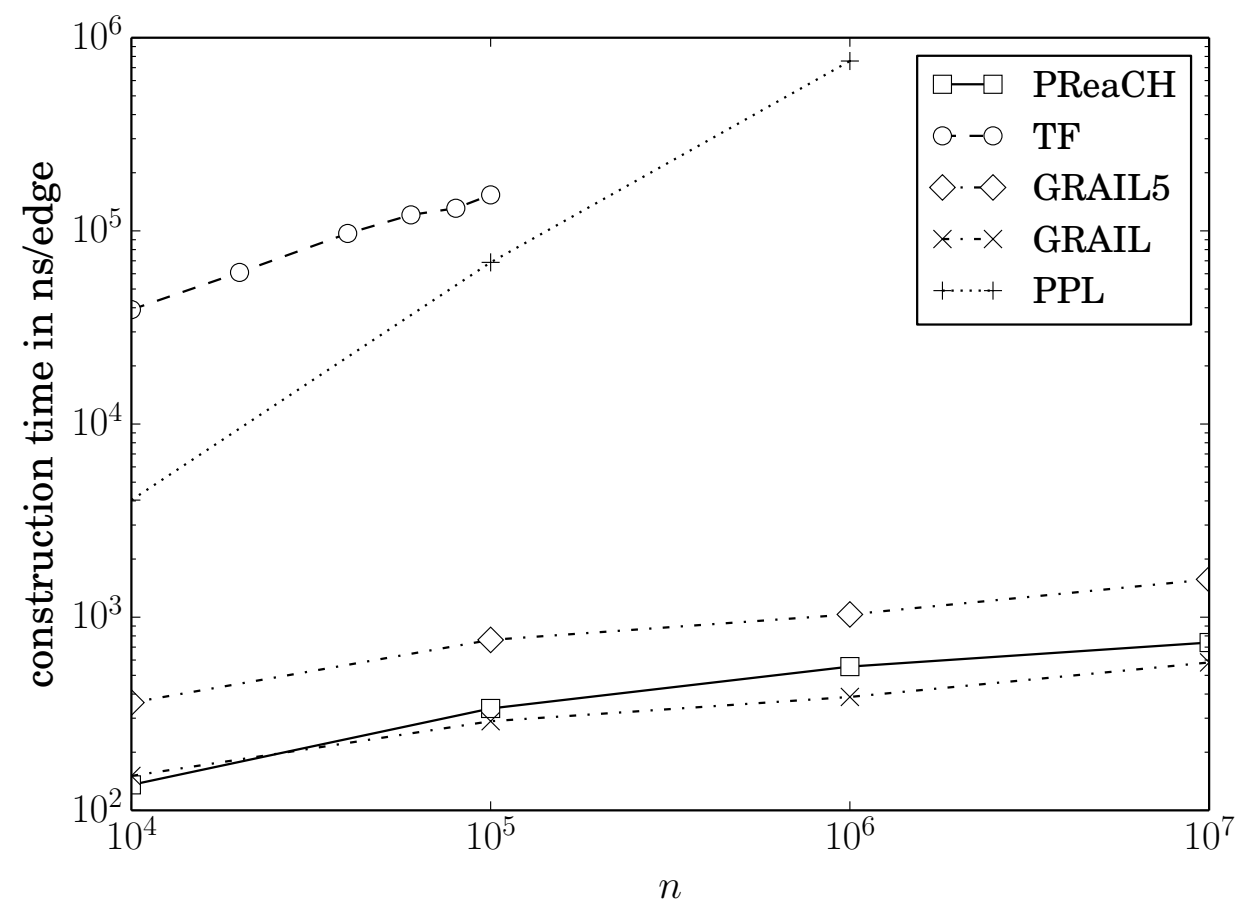

Figure 6: Construction time per edge for random DAGs with $n=10^{4} \ldots 10^{7}, m / n=8$

somewhat more stable. In particular, its maximal query time for (among the 100000 positive and 100000 negative queries tried) is two orders of magnitude smaller than GRAIL5. The differences are less pronounced in the other instances, but PReaCH still clearly outperforms GRAIL5 also with respect to the distribution of query times. The difference is particularly big for positive queries. For instances citeseerx and wiki-Talk PPL is not only better on average but also shows much less fluctuations in query time. Recall that for instance random10m10x PPL did not finish preprocessing.

\section{Conclusion}

The question about the best reachability index is very complex since it depends (at least) on the criteria query time, construction time, space, and the characteristics of the used instances. However, PReaCH is a serious candidate in many situations. It has near linear preprocessing time with small constant factors and needs linear space. Among the methods with these "light-weight" properties (like GRAIL [34]) PReaCH is clearly the fastest with respect to query time. Other methods like TF [6] and PPL [32] are considerably faster for some instances but pay with larger preprocessing time and higher or less predictable space consumption.

Another advantage of PReaCH is that it seems to be more easy to adapt this approach 


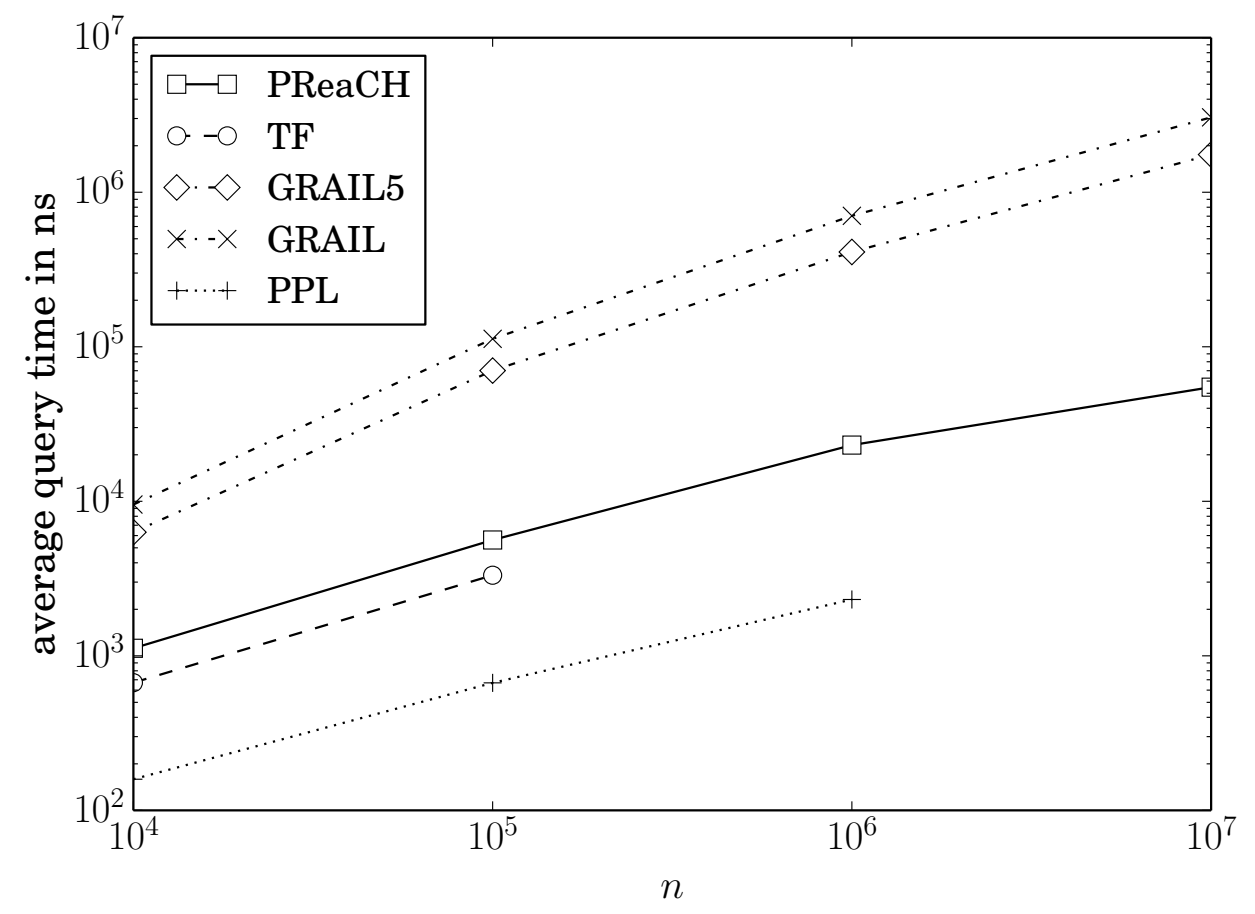

Figure 7: Average query time for random DAGs with $n=10^{4} \ldots 10^{7}, m / n=8$

to actually compute paths for positive queries. RCHs explicitly generate a path that, due to the absence of shortcuts in our implementation, need not even be unpacked. Pruning rules involving empty intervals and topological levels are no problem since they are not applied on the path. The only problem are pruning rules involving full intervals. The full interval $\phi(v) . . \hat{\phi}(v)$ can be handled by explicitly storing parent pointers for the DFS tree: if we stop the search because $t \in \phi(v) . . \hat{\phi}(v)$ we can reconstruct the path from $v$ to $t$ by following parent pointers from $t$. The full interval $\phi\left(p_{\text {tree }}(v)\right) . . \hat{\phi}\left(p_{\text {tree }}(v)\right)$ is more complicated. However, by storing $p_{\text {tree }}(v)$ explicitly we can search for the edge $(v, w)$ defining $p_{\text {tree }}(v)$ among the edges leaving $v$. We follow these edges until we reach $p_{\text {tree }}(v)$. From there we can backtrace parent pointers from $t$. Analogous strategies work for the backward part of the search.

The constant factor in the space consumption of PReaCH might be too expensive in some applications. However, we can derive very space efficient reachability indices from PReaCH also. For example using a variant of RCHs, we only need to store the graph itself plus a few bits telling where to split the edges stored with a node between forward and backward search space. Except for the Kronecker graphs, all the instances given in Table 1 need at most eight bits for representing a topological level. Hence, with two bytes per node one can support pruning with topological levels additionally.

From a conceptual point of view it is interesting that the usefulness of RCHs underlines that analogies between speedup techniques for route planning and reachability indices deserve further attention. 


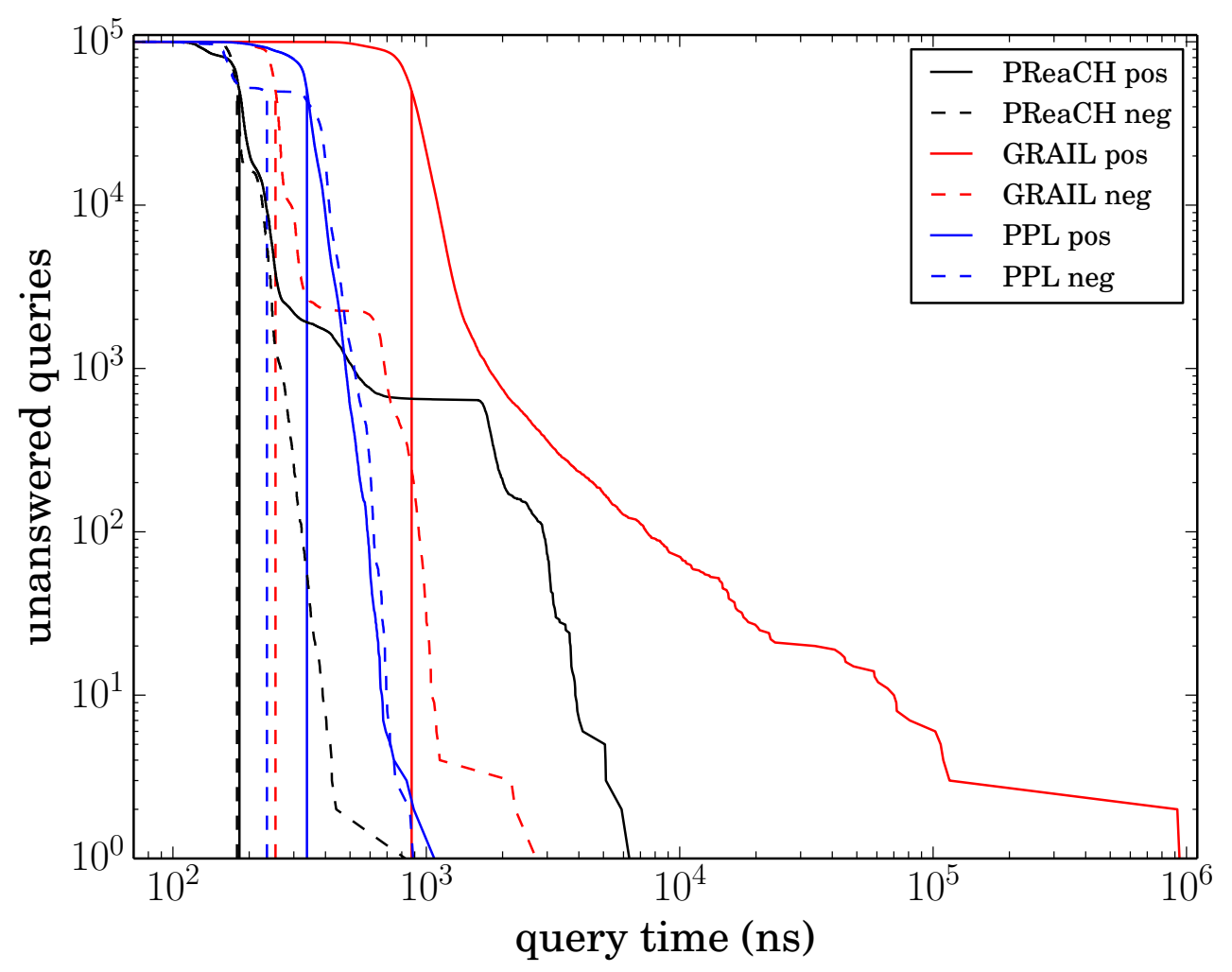

Figure 8: Query time distribution of 100000 queries on wiki-Talk.

\section{Future Work}

Our result suggest several promising avenues for further research. Staying close to PReaCH, we can try to trade time for space by using several DFS orderings simultaneously for pruning the search. We can trade query time for preprocessing time by performing several DFS searches (with random tie breaking) and only use the best one for actually storing the index. Judging what "the best" is could be based on performing queries for a random sample. The same idea can be applied to the contraction hierarchy. More interesting would be more clever heuristics to find good DFS-orderings and RCH-orderings. For example, for DFS we could better approximate the tree sizes by actually performing complete DFS explorations from all sources before deciding what the first tree is going to be. For RCH-ordering, the simple, static priority function based on degree seems only like a very first attempt 5 For example, CHs for route planning [12] use an estimate of the (unpruned) search space size as an important term in the priority function.

Besides compressing topological levels as mentioned above, we can also compress the data derived from DFS traversal. It suffices to store $\hat{\phi}(v)-\phi(v)$ rather than $\hat{\phi}(v)$ which will be small for most nodes. We could for example represent only values between 0 and 254

\footnotetext{
${ }^{5}$ We did try ordering nodes by their degree in the remaining graph rather than by their degree in the input graph. However, this did not yield improvement in query time justifying the overhead during construction.
} 


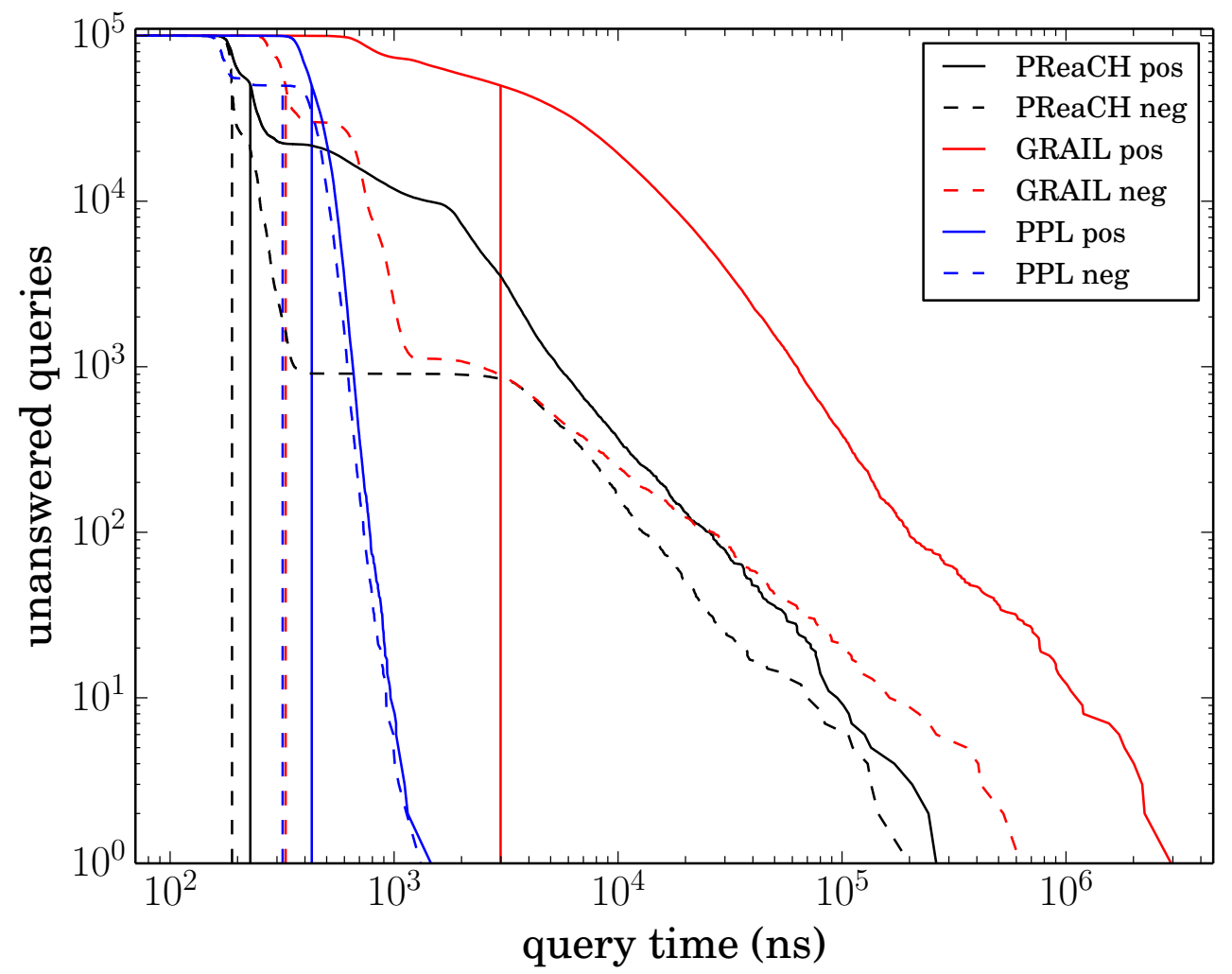

Figure 9: Query time distribution of 100000 queries on citeseerx.

directly using 255 as an escape value indicating that the true value can be found in a small hash table. We have already mentioned that at the cost of an additional indirection, the values $\phi\left(p_{\text {tree }}(v)\right)$ and $\hat{\phi}\left(p_{\text {tree }}(v)\right)$ do not need to be stored when we store $p_{\text {tree }}(v)$. All these measures combined would reduce the space requirement by about one third.

When scaling to even larger graphs, we would like to parallelize preprocessing. A certain degree of 'easy' parallelization is available by computing the $\mathrm{RCH}$, topological levels and DFS based information independently. Moreover we can perform multiple DFS - using all its information or only the one that works best. For graphs with not too many topological levels, finding these levels can be done by peeling them off in parallel. A similar strategy works for CHs (see also [12]) for route planning. There is also intensive work on finding SCCs in parallel (e.g., [13]). For finding full intervals as in Section 3.3 we could also use any preorder numbering of any spanning forest of the graph, e.g., based on BFS. Only for the empty intervals we would need a replacement for DFS with similarly useful properties.

Exploiting another analogy to route planning [1], we could use RCHs to derive a fast 2-hop-labelling scheme for reachability - simply use the $\mathrm{CH}$-search spaces from each node as its labels. Note that this could even be done in a lazy fashion - start with RCHs (or full $\mathrm{PReaCH}$ ) but whenever a node is queried, cache the discovered search space. Combining this with the ideas in PPL [32] like pruning search spaces or using paths rather than nodes 


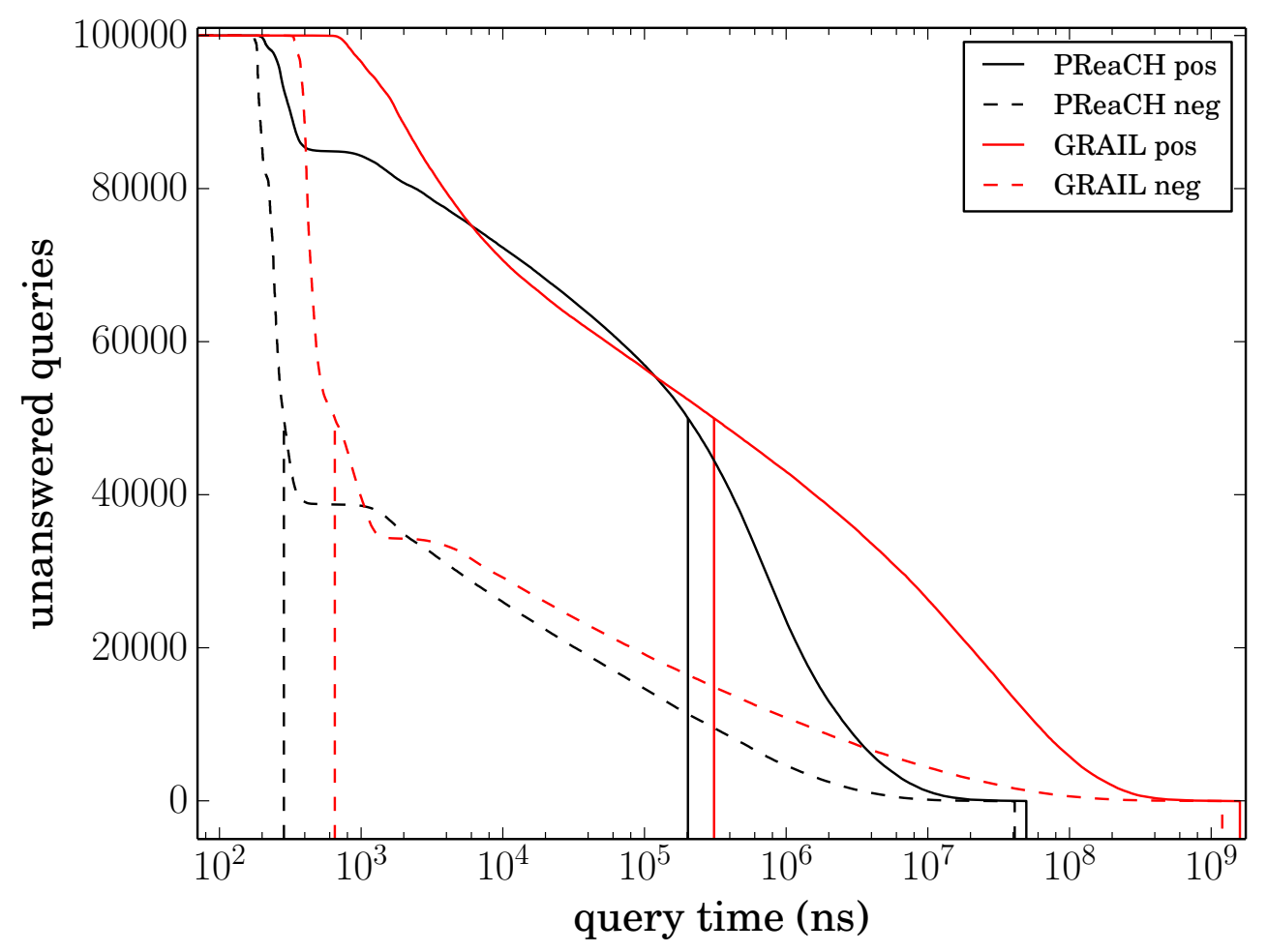

Figure 10: Query time distribution of 100000 queries on random10m10x.

in labels could give additional improvements.

\section{References}

[1] I. Abraham, D. Delling, A. V. Goldberg, and R. F. F. Werneck. A hub-based labeling algorithm for shortest paths in road networks. In 10th International Symposium on Experimental Algorithms, volume 6630 of Lecture Notes in Computer Science, pages 230-241, 2011.

[2] H. Bast, D. Delling, A. G. and Matthias Müller-Hannemann, T. Pajor, P. S. D. Wagner, and R. F. Werneck. Route planning in transportation networks. Technical Report MSR-TR-2014-4, Microsoft Research, 2014.

[3] H. Bast, S. Funke, P. Sanders, and D. Schultes. Fast routing in road networks with transit nodes. Science, 316(5824):566, 2007.

[4] L. Chen, A. Gupta, and M. E. Kurul. Stack-based algorithms for pattern matching on dags. In Proceedings of the 31st International Conference on Very Large Data Bases (VLDB), pages 493-504. VLDB Endowment, 2005. 
[5] Y. Chen and Y. Chen. An efficient algorithm for answering graph reachability queries. In Proceedings of the 24th International Conference on Data Engineering (ICDE), pages 893-902. IEEE, 2008.

[6] J. Cheng, S. Huang, H. Wu, and A. W.-C. Fu. TF-Label: A topological-folding labeling scheme for reachability querying in a large graph. 2013.

[7] J. Cheng, J. X. Yu, X. Lin, H. Wang, and S. Y. Philip. Fast computation of reachability labeling for large graphs. In Proceedings of the 10th International Conference on Extending Database Technology (EDBT), pages 961-979. Springer, 2006.

[8] J. Cheng, J. X. Yu, X. Lin, H. Wang, and P. S. Yu. Fast computing reachability labelings for large graphs with high compression rate. In Proceedings of the 11th International Conference on Extending Database Technology (EDBT), pages 193-204. ACM, 2008.

[9] E. Cohen, E. Halperin, H. Kaplan, and U. Zwick. Reachability and distance queries via 2-hop labels. SIAM Journal on Computing, 32(5):1338-1355, 2003.

[10] T. H. Cormen, C. E. Leiserson, and R. L. Rivest. Introduction to Algorithms. McGrawHill, 1990.

[11] A. Deutsch, M. Fernandez, D. Florescu, A. Levy, and D. Suciu. A query language for XML. Computer networks, 31(11):1155-1169, 1999.

[12] R. Geisberger, P. Sanders, D. Schultes, and C. Vetter. Exact routing in large road networks using contraction hierarchies. Transportation Science, 46(3):388-404, 2012.

[13] S. Hong, N. C. Rodia, and K. Olukotun. On fast parallel detection of strongly connected components (scc) in small-world graphs. In Proceedings of SC13: International Conference for High Performance Computing, Networking, Storage and Analysis, SC '13, pages 92:1-92:11, New York, NY, USA, 2013. ACM.

[14] A. M. Huerta, H. Salgado, D. Thieffry, and J. Collado-Vides. Regulondb: a database on transcriptional regulation in escherichia coli. Nucleic Acids Research, 26(1):55-59, 1998.

[15] H. V. Jagadish. A compression technique to materialize transitive closure. ACM Trans. Database Syst., 15(4):558-598, Dec. 1990.

[16] R. Jin, N. Ruan, S. Dey, and J. Y. Xu. SCARAB: Scaling reachability computation on large graphs. In Proceedings of the 2012 ACM SIGMOD International Conference on Management of Data, pages 169-180. ACM, 2012.

[17] R. Jin, N. Ruan, Y. Xiang, and H. Wang. Path-tree: An efficient reachability indexing scheme for large directed graphs. ACM Transactions on Database Systems (TODS), 36(1):7, 2011. 
[18] R. Jin and G. Wang. Simple, fast, and scalable reachability oracle. In PVLDB, volume 14, pages 1978-1989, 2013.

[19] R. Jin, Y. Xiang, N. Ruan, and D. Fuhry. 3-hop: A high-compression indexing scheme for reachability query. In Proceedings of the 2009 ACM SIGMOD International Conference on Management of Data, pages 813-826. ACM, 2009.

[20] R. Jin, Y. Xiang, N. Ruan, and H. Wang. Efficiently answering reachability queries on very large directed graphs. In Proceedings of the 2008 ACM SIGMOD International Conference on Management of Data, pages 595-608. ACM, 2008.

[21] M. Krummenacker, S. Paley, L. Mueller, T. Yan, and P. D. Karp. Querying and computing with BioCyc databases. Bioinformatics, 21(16):3454-3455, 2005.

[22] O. Lassila and R. R. Swick. Resource description framework (RDF) model and syntax specification. Technical report, W3C, 1999.

[23] K. Mehlhorn and S. Näher. Bounded ordered dictionaries in $O(\log \log N)$ time and $O(n)$ space. Information Processing Letters, 35(4):183-189, 1990.

[24] R. C. Murphy, K. B. Wheeler, B. W. Barrett, and J. A. Ang. Introducing the graph 500. Cray Users Group (CUG), 2010.

[25] P. Sanders. Fast priority queues for cached memory. ACM Journal of Experimental Algorithmics, 5, 2000.

[26] R. Schenkel, A. Theobald, and G. Weikum. HOPI: An efficient connection index for complex XML document collections. In Proceedings of the 9th International Conference on Extending Database Technology (EDBT), pages 237-255, 2004.

[27] D. Schultes and P. Sanders. Dynamic highway-node routing. In 6th Workshop on Experimental Algorithms (WEA), volume 4525 of LNCS, pages 66-79. Springer, 2007.

[28] S. Trißl and U. Leser. Fast and practical indexing and querying of very large graphs. In Proceedings of the 2007 ACM SIGMOD International Conference on Management of Data, pages 845-856. ACM, 2007.

[29] P. van Emde Boas. Preserving order in a forest in less than logarithmic time. Information Processing Letters, 6(3):80-82, 1977.

[30] S. J. van Schaik and O. de Moor. A memory efficient reachability data structure through bit vector compression. In Proceedings of the 2011 ACM SIGMOD International Conference on Management of Data, pages 913-924. ACM, 2011.

[31] H. Wang, H. He, J. Yang, P. S. Yu, and J. X. Yu. Dual labeling: Answering graph reachability queries in constant time. In Proceedings of the 22nd International Conference on Data Engineering (ICDE), pages 75-75. IEEE, 2006. 
[32] Y. Yano, T. Akiba, Y. Iwata, and Y. Yoshida. Fast and scalable reachability queries on graphs by pruned labeling with landmarks and paths. In 22nd ACM Conference on information and Knowledge Management (CIKM), pages 1601-1606. ACM, 2013.

[33] H. Yildirim, V. Chaoji, and M. J. Zaki. GRAIL: Scalable reachability index for large graphs. Proceedings of the VLDB Endowment, 3(1-2):276-284, 2010.

[34] H. Yıldırım, V. Chaoji, and M. J. Zaki. Grail: a scalable index for reachability queries in very large graphs. VLDB Journal, 21(4):509-534, 2012. 